\title{
Remission of Heart Failure Through Endoluminal Repair of Femoral Arteriovenous Fistula with the Use of a Covered Stent
}

\author{
Antenor Portela, Raldir Bastos, Benjamim Pessoa, Renato Duarte, Marcos Medeiros, Jayro Paiva
}

Teresina, PI - Brazil

\begin{abstract}
We report the case of a 21-year-old male with highoutput heart failure due to a femoral arteriovenous fistula caused by a firearm wound. A new balloon expandable stent covered with polytetrafluorethylene was implanted in the artery to occlude the arteriovenous fistula. The fistula was immediately occluded and the artery remained patent. On the following day, the patient felt much better, with no symptoms of heartfailure. Additional follow-up is required to assure the usefulness of this less invasive procedure in the treatment of arteriovenous fistulas.
\end{abstract}

Peripheral arteriovenous fistulas caused by vascular trauma are rare and are difficult to repair surgically ${ }^{1}$. Their recognition and repair are mandatory to avoid local and systemic complications, such as ischemia and ulceration of the limbs and congestive heart failure ${ }^{2,3}$. Surgical repair of arteriovenous fistulas is the traditional treatment. In recent years, percutaneous treatment has been used more and more ${ }^{3}$. We report the case of a femoral arteriovenous fistula in a young male, who had a firearm wound in his left thigh causing high-output congestive heart failure. Endoluminal repair of the femoral arteriovenous fistula was performed with a metallic prosthesis covered with polytetrafluorethylene (PTFE).

\section{Case report}

A 21-year-old male was admitted to the emergency department complaining of dyspnea and tachycardic palpitations one month after suffering a firearm wound in his left thigh.

On physical examination, the patient was tachypneic,

Hospital São Marcos - Teresina

Mailing address: Antenor Portela - Rua Des. Pires de Castro, 584 - Sul - 64001-280

- Teresina, PI, Brazil

English version by Stela Maris C. Gandour tachycardic (120bpm), with paleness (++/4) in mucosas and skin, wide pulses, his blood pressure being 140/60mmHg. A pulsing mass was palpated in the middle third of his thigh with local elevation of the temperature, where a regurgitating murmur was present. Heart auscultation showed cardiac sounds of normal intensity, arrhythmic, tachycardic, and with no murmurs. Pulmonary auscultation was within the normal range. The electrocardiogram showed signs of left ventricular hypertrophy, supraventricular extrasystoles, and sinus tachycardia. Doppler echocardiogram showed a mild enlargement in the cavitary diameters, and a cardiac output of $10 \mathrm{~L} / \mathrm{min}$. A peripheral arteriography was performed, depicting the extension and location of the arteriovenous fistula in the middle third of the left thigh, measuring approximately $12 \mathrm{~mm}$ of extension (fig. 1).

In view of the technical difficulties of the conventional surgical treatment with the possibility of venous vascular lesion, bleeding, and difficulty of access, we chose the percutaneous treatment.

We punctured the right femoral artery, implanted a 9F valvate introducer, inserted a Simmons 6F catheter, which was manipulated to the contralateral superficial femoral artery. An arteriography was performed and showed a highoutput femoral arteriovenous fistula in the middle third of the left thigh, measuring about $12 \mathrm{~mm}$. A 0.35 ' exchange guidewire with $260 \mathrm{~cm}$ of length was placed through the catheter. The catheter was withdrawn and a peripheral JOSTENT Graft (standard version Jomed) of $38 \mathrm{~mm}$ of length was mounted on a balloon of peripheral angioplasty with $8 \times 40 \mathrm{~mm}$. This attempt of implanting the stent through the contralateral femoral artery was not very successful because the stent was dislocated from the balloon when we tried to pass from the right to the left iliac artery at the level of the distal aorta. We then had to pull the stent and withdraw it by arteriotomy. After that, we dissected the left femoral artery and proceeded to an anterograde puncture with direct view, inserting the $9 \mathrm{~F}$ introducer and directing it to the fistula. The stent was then successfully implanted in the region 


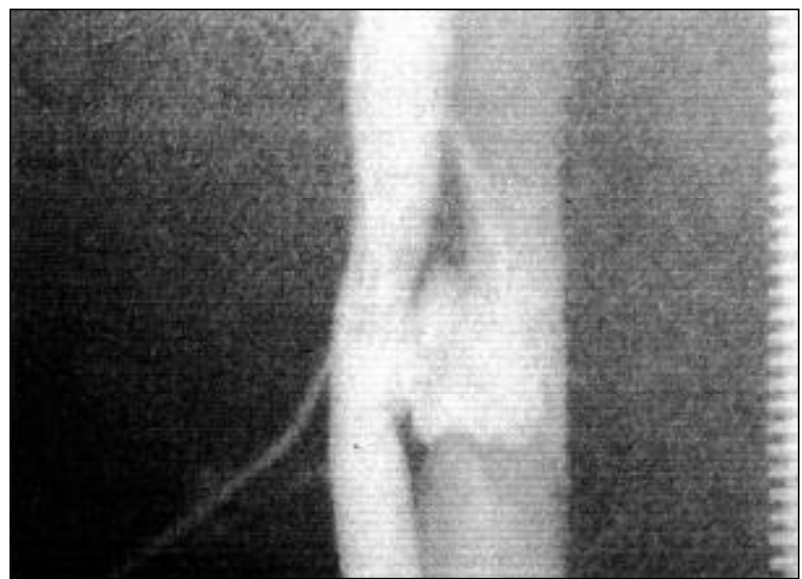

Fig. 1 - Left femoro-femoral arteriovenous fistula

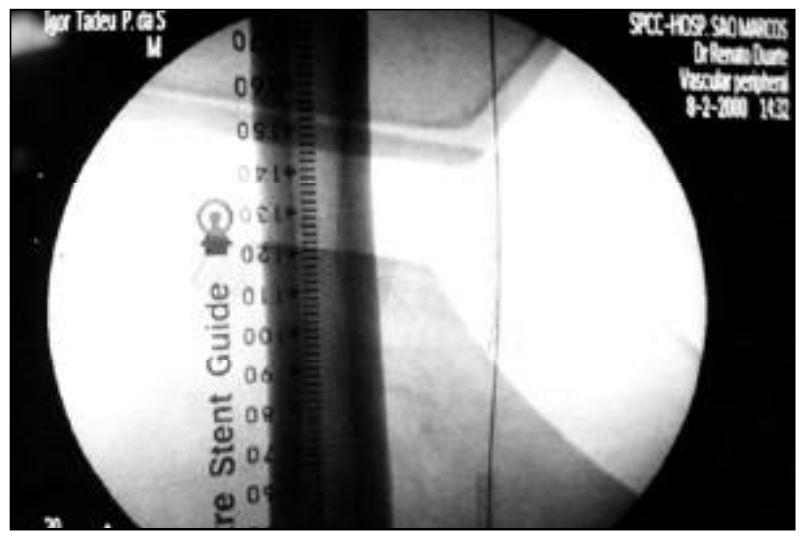

Fig. 2 - Stent-graft implanted in the fístula.

of the arteriovenous fistula (fig. 2). Confirmation of the optimal implantation and occlusion of the fistula was performed by injection of contrast medium through the introducer (fig. 3). The patient required general anesthesia because he was agitated at the moment of the procedure. On the following day, the patient was well, with no complaints. His hospital evolution was uneventful. Seventy-five days after the procedure, in an ambulatory visit, the patient had no complaints of heart failure. On physical examination, the lower limb showed a normal appearance and no murmur, the peripheral pulses were full and symmetric, and no evidence of thrombosis in the stent existed.

\section{Discussion}

Acquired arteriovenous fistulas are most often caused

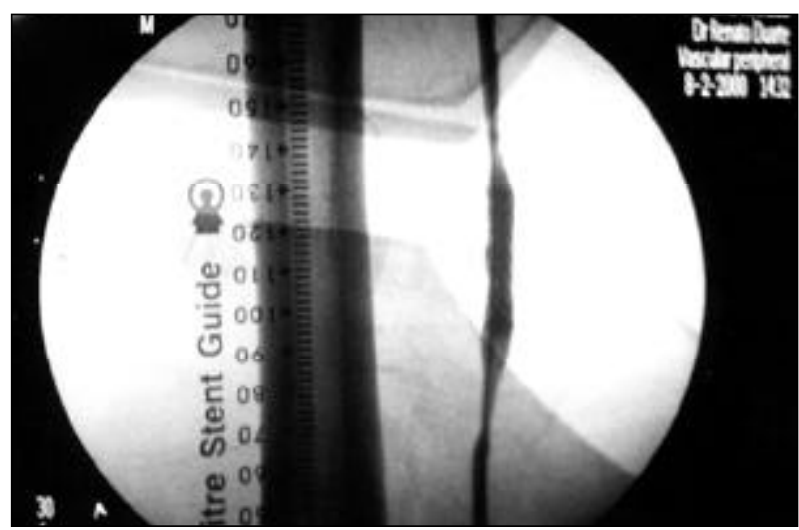

Fig. 3 - Arteriography after stent implantation showing occlusion of the fistula.

by firearm wounds, and even though they may occur in any part of the body, they are most frequently found in the thi$\mathrm{ghs}^{4}$. Surgical repair with excision is usually recommended in fistulas developing after a trauma.

The condition of high cardiac output associated with these fistulas depends on the size of the communication and the magnitude of the reduction in systemic vascular resistance $^{5}$. Another factor that also contributes for the development of heart failure is the sudden appearance of the lesion, such as in wounds caused by firearm.

Even though the traditional surgical repair has satisfactory results ${ }^{1}$, a less invasive treatment with implantation of covered stents is being performed more and more ${ }^{6,7}$.

The peripheral JOSTENT Graft is a new covered stent that has been used for treating aneurysms, pseudoaneurys$\mathrm{ms}$, and peripheral fistulas ${ }^{3,8}$. This prosthesis consists of 2 metallic stents with a layer of polytetrafluorethylene between the 2 stent bodies. We were not able to implant the stent percutaneously by contralateral femoral puncture, because the flexibility of the stent was not enough to allow crossing the bifurcation of the distal aorta. This may have happened because we were navigating with no vascular sheath for the prosthesis, using only the guidewire. The prosthesis was effective for occluding the fistula.

Late thrombosis of the prosthesis is a complication reported and requires longer follow-up to be detected, because it is reported at the end of the first year, and it is more common in patients with a poor distal blood flow ${ }^{9}$. Our patient had no sign of complication on physical examination $75 \mathrm{da}-$ ys after the procedure. Therefore, endoluminal repair of peripheral arteriovenous fistula with covered stent was a therapeutical alternative for vascular surgery in this case, eliminating the symptoms of heart failure ${ }^{7,8,10}$.

\section{References}

1. Khoury G, Stein R, Nabbout G, Jabbour-Khoury S, Fahl M. Traumatic arteriovenous fistula: "the Lebanese war experience". Eur J Vasc Surg 1994; 8: $171-3$.

2. Uflacker R, Elliott BM. Percutaneous endoluminal stent-graft repair of an old traumatic femoral arteriovenous fistula. Cardiovasc Intervent Radiol 1996; 19 : 120-2.

3. Ruebben A, Rossaro D, Savio S, Tettoni S, Muratore PL, Rabbia C. Initial Clinical Experience with a new stent-graft. Cardiovasc Intervent Radiol 1998; 21(suppl 1). 
4. Dorney ER. Peripheral AV fistula of fifty-seven years duration with refractory heart failure. Am Heart J 1957; 54: 778.

5. Flaim SF, Mintees WJ, Nellis SH, Clark DP. Chronic arteriovenous shunt: Evaluation of a model for heart failure in the rat. Am J Physiol 1979; 236: H398

6. Marin ML, Veith FJ, Panetta TF, et al. Percutaneous transfemoral insertion of a sented graft to repair a traumatic femoral arteriovenous fistula. J Vasc Surg 1993; 18: 299-302.

7. Thalhammer C, Kirchherr AS, Uhlich F, Waigand J, Gross CM. Postcatheterization pseudoaneurisms and arteriovenous fistulas: repair with percutaneous implantation of endovascular covered stents. Radiology 2000; 214: 127-31.

8. BurgerT, MeyerF, Tautenhahn J, Halloul Z, Fahlke J. Percutaneous treatment of rare iatrogenic arteriovenous fistulas of the lower limbs. Int Surg 1998; 83: 198-201.

9. Waigand J, Uhlich F, Gross CM, Thalhammer C, Dietz R. Arteriovenous fistulas after invasive vascular procedures. Catheter Cardiovasc Interv 1999; 47: 165-6.

10. Parodi JC, Schanholz C, Ferreira LM, Beregan J. Endovascular stent-graft treatment of traumatic arterial lesions. Ann Vasc Surg 1999; 13: 121-9. 